\title{
Recomendaciones del Ministerio de Salud de la Argentina para el cuidado de la salud en adolescentes LGBTI
}

Argentine Ministry of Health recommendations on LGBTI adolescent's health care

Escobar JCE, Chiodi A. Ministerio de Salud de la Nación. 2017.

\section{Introducción}

La adolescencia es una etapa clásicamente conflictiva del ciclo vital. Implica importantes cambios físicos, intelectuales y afectivos, entre otros, que posicionan a quien atraviesa esta etapa en situaciones de vulnerabilidad especiales ${ }^{1}$

Si bien habitualmente el contacto con los sistemas de salud durante la adolescencia son menores que en la infancia ${ }^{2,3}$, éstos pueden proveer oportunidades importantes para la prevención y promoción de la salud ${ }^{4}$. La comunidad LGBTI (lesbiana, gay, bisexual, trans, intersex) en la adolescencia está expuesta a situaciones especiales de vulnerabilidad, como el bullying ${ }^{5}$, violencia intrafamiliar, discriminación y su consecuente afectación de la salud mental ${ }^{6,7}$. La ideación suicida es mayor en esta etapa del ciclo vital pero lo es aún más en adolescentes de la comunidad LGBT|6,8.

Esta guía desarrollada por el Ministerio de Salud de la Nación Argentina ${ }^{9}$ brinda un marco conceptual para aquellos que no están familiarizados con los conceptos más modernos de sexualidad y género, enfocado al grupo de adolescentes. Consecuentemente, provee lineamientos sugeridos para la atención integral de los adolescentes LGBTI, como así también material para la sensibilización y educación en relación a los mencionados conceptos del marco conceptual.

En la siguiente sección resumimos los principales capítulos del documento.

\section{Resumen de aspectos destacados}

Capítulo I: Conceptos y herramientas teóricas. En este capítulo se invita a la reflexión de los profesionales de salud acerca de sus conceptos previos sobre la temática LGBTI: el dinamismo de la sexualidad, los problemas de identificar a la heterosexualidad como "la normalidad", la importancia de diferenciar la identidad de género de la orientación sexual, cómo la identidad de género no necesariamente oscila en el binario masculino/femenino (por ejemplo, en los intersex) y cómo la "expresión de género" (cómo se "muestra" la identidad de género) está fuertemente condicionada por factores socioculturales. Se explican los conceptos de cis-género (personas que identifican su género con el asignado al nacer) y transgénero. Este capítulo finaliza listando la variedad de formas de discriminación en relación a la sexualidad y cómo ésta puede afectar no solamente a los miembros de la comunidad LGBTI.

Capítulo II: Salud y Adolescencias LGBTI. Se inicia con una descripción de las vulnerabilidades específicas de los adolescentes LGBTI: bullying o acoso escolar, violencia en la familia de origen o con la pareja, problemas de salud mental (depresión, ansiedad, pánico), infecciones de transmisión sexual (especialmente en relación a la clandestinidad de las prácticas sexuales por condicionantes sociales) y consumo problemático de sustancias. A continuación se hace una descripción de cuatro barreras clave en relación a actitudes de los profesionales de la salud para la atención en salud de esta comunidad (ver Tabla 1). También se enumeran otras barreras adicionales como ser: mitos, desinformación, discriminación, omisión y patologización para subgrupos específicos dentro del colectivo LGBTI. El capítulo cierra con 17 recomendaciones básicas para una adecuada interacción con los miembros del colectivo LGBTI.

Capítulo III: Atención clínica a adolescentes LGBTI. En este capítulo se detallan todos los elementos de la consulta periódica de salud de los adolescentes. Se provee una guía de preguntas orientativas para indagar sobre el contexto familiar, social, económico, laboral, con una anamnesis completa y detallada acerca de los hábitos, sexualidad y actividades sociales, aclarando que no se pretende recabar toda esa información en una única consulta. La guía sugiere realizar auscultación cardíaca, medir peso, talla, índice de masa corporal, toma de tensión arterial, inspección bucodental, agudeza visual y evaluación del estadío de Tanner. Como estudios complementarios sugiere: hemograma, glucemia (ante factores de riesgo para diabetes), dosaje de lípidos a los 17 años, testeo para sífilis y VIH en individuos sexualmente activos y electrocardiograma (única vez). También sugiere discutir la presencia de depresión, consumo problemático de sustancias y prevención de accidentes (lesiones no intencionales). Sugiere la consejería en términos de anticoncepción y en los casos que sea necesario, sobre interrupción legal del embarazo (ILE). Sugiere revisar el calendario de inmunización según las recomendaciones actuales del Ministerio de Salud. Un aspecto distintivo de este capítulo es las consideraciones que se realizan en cuanto a la consejería que puede proveer el personal de salud en relación a la "salida del closet" (declarar voluntaria y públicamente la orientación sexual). El documento brinda alguna guía sobre la salud de personas trans, explicitando que los adolescentes a partir de los 16 años podrían comenzar tratamientos hormonales, y aún antes, medidas para visibilizar su identidad de género. En cuanto al PAP, la guía explica que los varones trans que tengan cuello uterino, son pasibles al rastreo dentro del marco etáreo que recomienda el Ministerio de Salud (a partir de los 25 años). La guía recomienda la realización del PAP anal en personas VIH positivas, personas con lesiones por HPV, personas que practican sexo anal receptivo e inmunosuprimidos. Al final del capítulo existen algunas recomendaciones actitudinales que se resumen en la Tabla 2.

Capítulo IV: contiene una serie de actividades y dinámicas de trabajo con adolescentes para la sensibilización de conceptos relacionados a la diversidad sexual, expresión e identidad de género.

Capítulo V: contiene una compilación de material audiovisual sugerido como soporte a las actividades prácticas y teóricas propuestas por la guía.

Capítulo VI: contiene una referencia para organizaciones e instituciones que apoyan al ejercicio de los derechos de la comunidad LGBTI.

Capítulo VII: recopila el marco legal nacional, regional e internacional que abordan los derechos de la comunidad LGBTI. 
Tabla 1. Barreras para el acceso a los servicios de salud para los adolescentes LGBTI.

\begin{tabular}{l|l|l} 
Neutralización & \multicolumn{1}{|c}{ ¿Qué implica? (Premisa) } & \multicolumn{1}{c}{ ¿Por qué es una barrera? } \\
\hline Evitación & $\begin{array}{l}\text { "La orientación sexual e identidad de género son irrelevantes para la } \\
\text { provisión de servicios de salud" }\end{array}$ & $\begin{array}{l}\text { Es una forma de discriminación que invisibiliza las especificidades de } \\
\text { la persona }\end{array}$ \\
\hline Segregación & "De eso no se habla"0 "ni sabría ni cómo hacerlo" & $\begin{array}{l}\text { Se pierden oportunidades de abordar cuestiones relativas a la } \\
\text { sexualidad y el género }\end{array}$ \\
\hline Exotización & "Ios LGBT suelen ser inestables, promiscuos... grupos de alto riesgo" & $\begin{array}{l}\text { Provocan una homogeneización y estigma, que no se centra en las } \\
\text { características individuales }\end{array}$ \\
\hline
\end{tabular}

Tabla 2. Recomendaciones en la atención de adolescentes LGBTI.

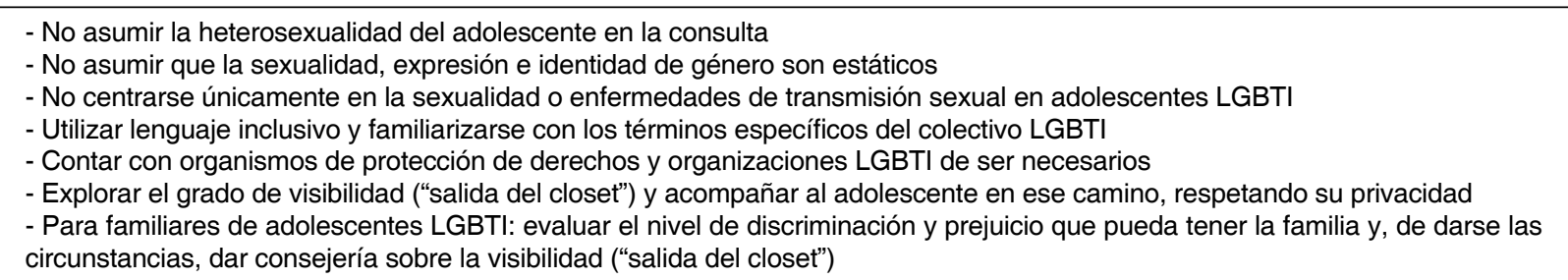

Nota: se seleccionaron algunas de las recomendaciones más relevantes de la guía que fueron resumidas por el autor de este artículo.

\section{Comentarios}

Este documento desarrollado por el Ministerio de Salud de Argentina es un aporte interesante para la integración de la atención de individuos LGBTI. Su enfoque en la adolescencia tiene particular interés debido a que, durante la misma, se suelen presentar las primeras experiencias sexuales de los individuos, como así también su expresión de género de adulto (si bien ésta puede ser explícita mucho antes). Éstas pueden fluctuar con el tiempo y durante este periodo de particular vulnerabilidad en la transición de la niñez a la adultez, puede ser sujeto a actitudes no integradoras (o discriminatorias) por parte de los profesionales de la salud. Es por eso que el mayor valor de esta guía, desde el punto de vista de su construcción teórica, es su fin sensibilizador para los profesionales de la salud. Otras guías en la región han tenido este abordaje desde la salud ${ }^{9,10}$, mientras que en otras regiones del mundo se han generado documentos con un propósito sensibilizador más global (intersectorial) ${ }^{11}$. Otro punto fuerte de la guía es la diversidad de recursos didácticos y audiovisuales que propone para lograr su objetivo educativo, descritos ampliamente en los capítulos IV y V; como así también la amplia participación de organizaciones de derechos humanos en la revisión externa del documento. Sin embargo, de manera similar a la "Guía de la salud integral de personas trans" que comentamos en esta misma revista ${ }^{12}$, esta guía sufre limitaciones similares: a) Existe una ausencia de una metodología sistemática para la búsqueda de las evidencias y posterior evaluación de su calidad, previo a la realización de las recomendaciones; b) El insuficiente detalle en las mismas como para que el médico ponga en práctica muchas de las intervenciones sugeridas (por ejemplo: intervalos de seguimiento, conductas ante hallazgos clínicos, etc.); c) No se especifican estrategias de implementación (más allá de lo educativo) ni de actualización; d) Es una guía extensa (120 páginas) lo que puede limitar su difusión al personal de salud, al no contar con un resumen ejecutivo. En el capítulo III se lista una serie de recomendaciones clínicas en adolescentes que no cuentan con amplio consenso o sustento científico (como el rastreo de dislipidemias en adolescentes o la realización de electrocardiograma en individuos asintomáticos) y que, además, pueden resultar altamente estigmatizantes (como la sugerencia controversial de realizar PAP anal rutinario en individuos que realizan sexo anal receptivo). Un aspecto positivo, sin embargo, es que la recomendación para el rastreo de cáncer de cuello de útero se encuentra consistentemente enlazada con otra guía del Ministerio, que sí se encuentra sustentada en evidencia científica ${ }^{13}$. Es importante destacar que una revisión de guías de la misma temática ha encontrado las mismas limitaciones a nivel internacional ${ }^{11}$.

Juan Victor Ariel Franco [ Servicio de Medicina Familiar y Comunitaria del Hospital Italiano de Buenos Aires juan.franco@hospitalitaliano.org.ar ]

Franco JVA. Guía de práctica clínica del Ministerio de Salud de la Nación Argentina sobre salud en adolescentes LGBTI. Evid Actual Pract Ambul 2018;21 (4):120-121. Resumido y comentado de: Escobar JCE, Chiodi A. Salud y adolescencias LGBTI. Herramientas de abordaje integral para equipos de salud. Ministerio de Salud de la Nación. 1 ra ed; Ciudad Autónoma de Buenos Aires. 2017. Disponible en URL: http://www.msal.gob.ar/images/stories/ bes/graficos/0000001080cnt-salud-adol-Igbti.pdf. (último acceso abril 10, 2018).

\section{Referencias}

1. Servicio de Medicina Familiar y Comunitaria del Hospital Italiano de Bueno Aires. Medicina Familiar y Práctica Ambulatoria. Médica Panamericana; 2016. https://www.medicapanamericana.com/Libros/Libro/5399/Medicina-Familiar-y-Practica-Ambulatoria.html. Accessed April 10, 2018.

2. Berra S, y col. Correlates of Use of Health Care Services by Children and Adolescents From 11 European Countries. Med Care. 2009;47(2):161-167. doi:10.1097/MLR.0b013e3181844e09 3. Irwin CE, y col. Preventive Care for Adolescents: Few Get Visits and Fewer Get Services. Pediatrics. 2009;123(4):e565-e572. doi:10.1542/peds.2008-2601

4. Solberg Ll, y col. Clinical Preventive Services for Adolescents. Am J Prev Med. 2009;37(5):445-454. doi:10.1016/j.amepre.2009.06.017

5. Friedman MS, y col. A Meta-Analysis of Disparities in Childhood Sexual Abuse, Parental Physical Abuse, and Peer Victimization Among Sexual Minority and Sexual Nonminority Individuals. Am J Public Health. 2011;101(8):1481-1494. doi:10.2105/AJPH.2009.190009

6. Marshal MP, y col. Suicidality and Depression Disparities Between Sexual Minority and Heterosexual Youth: A Meta-Analytic Review. J Adolesc Heal. 2011;49(2):115-123. doi:10.1016/j. jadohealth.2011.02.005

7. Plöderl M, Tremblay P. Mental health of sexual minorities. A systematic review. Int Rev Psychiatry. 2015;27(5):367-385. doi:10.3109/09540261.2015.1083949

7. Plöderl M, Tremblay P. Mental health of sexual minorities. A systematic review. Int Rev Psychiatry. 2015;27(5):367-385. doi:10.3109/09540261.2015.1083949
8. Jackman K, y col. Nonsuicidal self-injury among lesbian, gay, bisexual and transgender populations: an integrative review. J Clin Nurs. 2016;25(23-24):3438-3453. doi:10.1111/jocn.13236 9. CDC-USA. LGBT Youth. https://www.cdc.gov/lgbthealth/youth.htm.

10. MSP-Uruguay. Guía En Salud Sexual y Reproductiva. Capítulo: Diversidad Sexual. Programa Prioritario ITS/SIDA Programa Nacional de Salud de La Mujer y Género.; 2009. http:// www.msp.gub.uy/sites/default/files/archivos_adjuntos/Guias en SSySR - Capitulo Diversidad Sexual 2009.pdf. Accessed August 9, 2018.

11. McNair RP, Hegarty K. Guidelines for the primary care of lesbian, gay, and bisexual people: a systematic review. Ann Fam Med. 2010;8(6):533-541. doi:10.1370/afm.1173

12. Franco JVA, Chaves SC. Atención de la salud integral de personas trans. Evid Act Pr Ambul. 2016;19(1):25-26.

13. Ministerio de Salud - Argentina. Guía Programática Abreviada Para El Tamizaje de Cáncer Cervicouterino. http://www.msal.gob.ar/images/stories/bes/graficos/0000000433cnt-Guia Programatica_Abreviada_BAJA.pdf. Accessed August 9, 2018. 